A discrete time Markov chain model for a periodic inventory system with one-way substitution

Yannick Deflem and Inneke Van Nieuwenhuyse

DEPARTMENT OF DECISION SCIENCES AND INFORMATION MANAGEMENT (KBI) 


\title{
A Discrete Time Markov Chain Model for a Periodic Inventory System with One-Way Substitution
}

\author{
Yannick Deflem, Inneke Van Nieuwenhuyse \\ Research Center for Operations Management, Department of Decision Sciences and Information Management, \\ K.U.Leuven, Naamsestraat 69,3000 Leuven, Belgium \\ Yannick.deflem@econ.kuleuven.be, Inneke.vannieuwenhuyse@econ.kuleuven.be
}

\begin{abstract}
This paper studies the optimal design of an inventory system with "one-way substitution", in which a high-quality (and hence, more expensive) item fulfills its own demand and simultaneously acts as backup safety stock for the (cheaper) low-quality item. Through the use of a discrete time Markov model we analyze the effect of one-way substitution in a periodic inventory system with an $(R, S, S)$ or $(R, S)$ order policy, assuming backorders, zero replenishment leadtime and correlated demand. In more detail, the optimal inventory control parameters $(S$ and $s$ ) are determined in view of minimizing the expected total cost per period (i.e. sum of inventory holding costs, purchasing costs, backorder costs and adjustment costs). Numerical results show that the one-way substitution strategy can outperform both the "no pooling" (only product-specific stock is held, and demand can never be rerouted to stock of a different item) and "full pooling" strategies (implying that demand for a particular product type is always rerouted to the stock of the flexible product, and no product-specific stock is held)- provided the mix of dedicated and flexible inputs is chosen adequately - even when the cost premium for flexibility is significant. Furthermore, we can observe that decreasing the demand correlation results in rerouting more demand to the flexible product and because of the risk-pooling effect reduces the optimal expected total cost.
\end{abstract}

Keywords: inventory management, one-way substitution

\section{Introduction}

In many supply chains, mismatches between supply and demand are (at least partially) mitigated through the use of inventories. Inventories can be kept at different levels of the supply chain, including raw materials, components, semi-finished products and/or end items. Successful inventory management needs to balance the benefits of inventory (i.e., reducing lost sales) with the associated cost (which is typically reflected in the inventory holding cost). 
One way of reducing the cost associated with inventory is to pool the demands of multiple items on the same (flexible) item: provided that demands are not perfectly positively correlated, pooling multiple demands on the same item allows a reduction in the required amount of safety stock, and (hence) a reduction in inventory holding cost. This is referred to as "risk-pooling" or "statistical economies of scale" (Van Mieghem, 2008). However, it tends to come at a cost: this "flexibility cost" can boil down to a product cost premium (when the flexible item is inherently more expensive to manufacture or purchase) and/or an additional adjustment cost (when the item needs to undergo additional processing or transportation in order to make it "fit for use" when demand arises).

This observation has spurred research on so-called substitution systems ${ }^{1}$, in which a high-quality (and usually more expensive) item fulfills its own demand and simultaneously acts as backup safety stock for the (cheaper) low-quality item. Substitution flexibility can be obtained in a variety of ways, a.o. through the use of manufacturer-driven one-way substitution (Bassok et al. 1999, Rutten and Bertrand 1998), lateral transshipments (e.g. Robinson 1990, Herer et al. 2006) and tailored postponement (TibbenLembke and Bassok 2005). It offers a compromise between a setting with "full pooling" (implying that demand for a particular product type is always rerouted to the stock of the flexible product, and no product-specific stock is held) and "no pooling" (only product-specific stock is held, and demand can never be rerouted to stock of a different product).

In the literature, periodic review inventory systems with substitution flexibility have been predominantly studied by means of newsvendor models (e.g. Van Mieghem 1998, Hale et al. 2001). As such, the current models focus mainly on single-period settings, ignoring the impact of stock replenishments (and the associated replenishment leadtimes). The assumption of negligible leadtimes tends to be common also in multi-period settings (e.g. Hillier 2002, Van Mieghem and Rudi 2002). Determining the optimal inventory control parameters in systems with substitution is typically complex: demands are only "partially pooled" on the inventory of the substitute, and how much is pooled depends on the order policies of both the dedicated product and the substitute. In the literature, the optimal order-up-to levels are typically found using simulation optimization (e.g. Robinson 1990, Khouja et al. 1996, TibbenLembke and Bassok 2005).

In this paper, a discrete time Markov model (DTMC) is developed to investigate the optimal design of a 2 -item inventory system with one-way substitution in a multi period setting. The goal of this paper is to study the effect of one-way substitution in a $(R, s, S)$ inventory system, assuming zero replenishment leadtime, backorders and correlated demand. More specifically, we want to determine the optimal inventory control parameters for three strategies (one-way substitution, no pooling and full pooling), in

\footnotetext{
${ }^{1}$ This is also referred to as "tailored flexibility", "tailored pooling" (Chopra and Meindl 2007) or "tail-pooling" (Van Mieghem 2008).
} 
view of minimizing the expected total cost per review period (comprising expected adjustment costs, expected purchasing costs, expected inventory holding costs and expected shortage costs).

Section 2 describes the research problem in further detail. The DTMC approach for the three strategies is presented in Section 3. Section 4 discusses the results of a computational study, in which the three strategies are compared. Section 5 summarizes the main conclusions.

\section{Problem description}

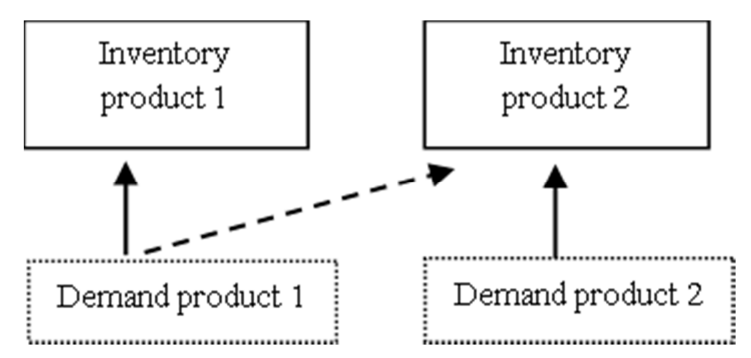

Figure 1: Two products with one-way substitution

Consider a setting with two different product types (Product 1 and Product 2) as in Figure 1. Demand $d_{i}$ for a specific product type $i$ is preferably satisfied by means of the corresponding (product-specific or dedicated) inventory (this is indicated by the solid arrows). Only when that inventory is out of stock, demand can be satisfied by a substitute. In the figure, it is assumed that demand for product 1 can be satisfied by inventory of product 2 (as indicated by the dashed arrow), and not vice versa. This is the essence of one-way substitution. This situation often arises naturally in real-life settings, e.g. when item 2 has higher quality or wider functionality than item 1.

We assume that the inventories of both products are managed according to an $(R, s, S)$ policy. At the end of every review period $R$, the decision maker allocates the available inventory to the observed demand according to the rules described above. Demand that cannot be met is backlogged. When, following this allocation, the inventory position of product $i$ is smaller than or equal to its reorder point $s_{i}$, an order $Q_{i}$ is placed such that the inventory position is raised to the order-up-to level $S_{i}$ (with $S_{i}>s_{i}$ ) (Chopra and Meindl 2007). Since the replenishment leadtime is assumed to be zero, orders are received immediately; consequently, the net inventory immediately rises to $S_{i}$ once an order for item $i$ has been placed. Note that the well-known $(R, S)$ policy is a special case of the $(R, s, S)$ policy with $s=S-1$ : an order is placed at the end of every review period provided the inventory position is smaller than $S$. Demands for both products are assumed to be discrete and finite random variables with a joint probability mass function $P_{D}\left(d_{1}, d_{2}\right)$, with $d_{i}$ the demand realization of product $i$. 
Table 1 gives an overview of the cost parameters and random variables which are represented in the objective function (expected total costs per period). The amount of demand rerouted to the substitute at the end of a review period is denoted by $Z$ (the dashed line in Figure 1) and incurs a unit adjustment cost $a$. Any leftover inventory or safety stock $I_{i}$ of product $i$ at the end of a review period incurs a unit holding $\operatorname{cost} h_{i}$. Demand of product $i$ that cannot be satisfied at the end of a review period is backlogged; this backlog is denoted by $B_{i}$ and is penalized at a unit shortage cost $p_{i}$. If a replenishment order for product $i$ is placed, a unit purchasing $\operatorname{cost} c_{i}$ is incurred. For any random variable $X$, the notation $\mathrm{E}[X]$ refers to the expected value of $X$.

\begin{tabular}{|l|l|}
\hline \multicolumn{2}{|l|}{ Cost parameters } \\
\hline$c_{i}$ & Purchasing cost per unit of product $i$ \\
\hline$p_{i}$ & Shortage cost per unit of unsatisfied demand of product $i$ at the end of a review period \\
\hline$h_{i}$ & Holding cost per unit of product $i$ left over at the end of a review period \\
\hline$a$ & Adjustment cost per unit of demand for product 1 satisfied by product 2 \\
\hline Random variables \\
\hline$Q_{i}$ & Replenishment order size of product $i$ \\
\hline$B_{i}$ & Amount of backorders of product $i$ incurred at the end of a review period \\
\hline$I_{i}$ & Leftover inventory or safety stock of product $i$ at the end of a review period \\
\hline$Z$ & Amount of demand rerouted to the substitute at the end of a review period \\
\hline$d_{i}$ & Period demand of product $i$ \\
\hline Decision parameters \\
\hline$S_{i}$ & Order-up-to level of product $i$ \\
\hline$s_{i}$ & Reorder point of product $i$ \\
\hline
\end{tabular}

Table 1: Notation

For a meaningful analysis, the following assumptions need to hold:

Assumption 1: $c_{2}-c_{1}+a>0$

The flexibility cost is strictly positive.

Assumption 2: $p_{1}+h_{2}>a+c_{2}-c_{1}$ 
In case of a shortage of product 1 and leftover inventory of product 2, it is more expensive to do nothing and incur $p_{1}+h_{2}$ than to use product 2 as a substitute and incur the associated flexibility cost (i.e., sum of adjustment cost and product cost premium).

Assumption 3: $h_{1}+a>h_{2}$

Transforming leftover inventory of product 2 into inventory of product 1 is never cost beneficial.

The optimal order policy in an inventory system with flexibility will heavily depend on the trade-off between the savings in safety inventory holding cost and the increase in flexibility cost incurred. As will be illustrated in Section 4.2, rerouting more demand to the flexible product (and hence pooling more demand on the flexible product) may reduce the total need for safety stock in the system (and the associated inventory holding cost) because of the risk-pooling effect. The extent of these holding cost savings depends highly on the correlation between the demands of the product and its substitute. At the same time however, the total flexibility cost incurred increases. This flexibility cost is reflected in the expected adjustment cost and/or the expected purchasing costs, while any decrease in customer service level is penalized through the expected shortage cost.

For any combination of $s_{i}$ and $S_{i}$ values, the expected total costs per period can be evaluated using a Discrete Time Markov Chain (DTMC), yielding the steady-state probabilities of the inventory positions of both product types.

\section{DTMC approach}

In this section, we present a discrete-time Markov model that allows to determine the expected total cost per period for the one-way substitution strategy, by evaluating $E\left[Q_{i}\right], E\left[I_{i}\right], E\left[B_{i}\right]$ and $E[Z]$ from the steady-state probabilities. With one-way substitution, the expected total cost per period $E[T C]$ is given by:

$E[T C]=\sum_{i=1}^{2}\left(c_{i} E\left[Q_{i}\right]+p_{i} E\left[B_{i}\right]+h_{i} E\left[I_{i}\right]\right)+a E[Z]$

The expected values $E\left[Q_{i}\right], E\left[I_{i}\right], E\left[B_{i}\right]$ and $E[Z]$ can be evaluated using a DTMC approach, where the state of the inventory system is defined by a two-dimensional state vector $(j, k)$. The first dimension $j$ represents the net inventory (i.e. on hand inventory minus number of backorders ${ }^{2}$ ) of product 1 at the end

\footnotetext{
${ }^{2}$ As the leadtime equals zero, there are no replenishment orders outstanding at any moment in time. Consequently, the net inventory is equal to the inventory position.
} 
of the review period, after demand has been allocated and before a replenishment order (if any) is placed. The second dimension $k$ represents the net inventory of product 2 in an analogous way.

As demand is discrete and finite (see Section 2), the discrete set of possible sates is finite. The net inventory for product $i(i=1,2)$ has an inherent upper bound $U B_{i}$ equal to the order-up-to level $S_{i}$ for the three strategies. Since demand is finite, the net inventory of product $i$ is also limited by a lower bound $L B_{i}$, as shown in Table 2 for the one-way substitution strategy. As evident from the table, the lower bounds are influenced by the reorder points $s_{i}$ and the maximum demands max $\left(d_{i}\right)$.

\begin{tabular}{|c|c|c|c|}
\hline & & $s_{2}+1 \geq \max \left(d_{2}\right)$ & $\boldsymbol{s}_{2}+\mathbf{1}<\max \left(\boldsymbol{d}_{2}\right)$ \\
\hline \multirow{2}{*}{$\begin{array}{l}s_{1}+1 \geq \\
\max \left(d_{1}\right)\end{array}$} & $L B_{1}$ & $s_{1}+1-\max \left(d_{1}\right)$ & $s_{1}+1-\max \left(d_{1}\right)$ \\
\hline & $L B_{2}$ & $s_{2}+1-\max \left(d_{2}\right)$ & $s_{2}+1-\max \left(d_{2}\right)$ \\
\hline \multirow{2}{*}{$\begin{array}{l}\boldsymbol{s}_{\mathbf{1}}+\mathbf{1} \\
<\max \left(\boldsymbol{d}_{\mathbf{1}}\right)\end{array}$} & $L B_{1}$ & $s_{1}+1-\max \left(d_{1}\right)+s_{2}+1-\max \left(d_{2}\right)$ & $s_{1}+1-\max \left(d_{1}\right)$ \\
\hline & $L B_{2}$ & 0 & $s_{2}+1-\max \left(d_{2}\right)$ \\
\hline
\end{tabular}

Table 2: Overview of lower bounds on net inventory with one-way substitution, for demand that is discrete and finite

In Section 3.1 we discuss the DTMC for the $(R, s, S)$ policy. The $(R, S)$ policy is a special case of the $(R, s, S)$ policy and is discussed in Section 3.2.

\subsection{DTMC for the $(R, s, S)$ policy}

Table 3 shows the transition probabilities from state $(j, k)$ to state $(l, m)$ for an $(R, s, S)$ policy with one- way substitution. As evident from the table, the transition probabilities depend on the current and next state $\left((j, k)\right.$ and $(l, m)$ respectively), on the reorder points $s_{i}$ and order-up-to levels $S_{i}$, and on the joint probability mass function $P_{D}$. Solving the Markov model through a system of linear equations, we obtain $\pi_{j, k}$, the steady-state probability of state $(j, k)$ (for all $j$ and $k$ ). The expected inventory of product 1 at the end of the review period can then be evaluated as:

$E\left[I_{1}\right]=\sum_{j=1}^{j=S_{1}}\left(j \sum_{k=L B_{2}}^{k=S_{2}} \pi_{j, k}\right)$

$\sum_{k=L B_{02}}^{k=S_{2}} \pi_{j, k}$ is the probability that the net inventory at the end of the period of product 1 is equal to $j$ units. Multiplying with $\mathrm{j}$ and adding over all strictly positive values of $j$ results in the expected inventory of product 1 at the end of the review period. The expression for $E\left[I_{2}\right]$ is similar:

$E\left[I_{2}\right]=\sum_{k=1}^{k=S_{2}}\left(k \sum_{j=L B_{1}}^{j=S_{1}} \pi_{j, k}\right)$ 


\begin{tabular}{|c|c|c|c|c|}
\hline \multirow{5}{*}{$j>s_{1}, k>s_{2}$} & From & To & Transition probability & For \\
\hline & $(j, k)$ & $(l, m)$ & $P_{D}(j-l, k-m)$ & $l>0$ \\
\hline & $(j, k)$ & $(l, m)$ & $P_{D}(j-l, k-m)$ & $l \leq 0, m<0$ \\
\hline & $(j, k)$ & $(0, m)$ & $\sum_{w=0}^{w=k-m} P_{D}(j+w, k-m-w)$ & $m \geq 0$ \\
\hline & $(j, k)$ & $(l, 0)$ & $\sum_{w=0}^{w=k} P_{D}(j-l+w, k-w)$ & $l<0$ \\
\hline \multirow{4}{*}{$j \leq s_{1}, k>s_{2}$} & $(j, k)$ & $(l, m)$ & $P_{D}\left(S_{I^{-}}-l, k-m\right)$ & $l>0$ \\
\hline & $(j, k)$ & $(l, m)$ & $P_{D}\left(S_{I^{-}}-l, k-m\right)$ & $l \leq 0, m<0$ \\
\hline & $(j, k)$ & $(0, m)$ & $\sum_{w=0}^{w=k-m} P_{D}\left(S_{1}+w, k-m-w\right)$ & $m \geq 0$ \\
\hline & $(j, k)$ & $(l, 0)$ & $\sum_{w=0}^{w=k} P_{D}\left(S_{1}-l+w, k-w\right)$ & $l<0$ \\
\hline \multirow{4}{*}{$j>s_{1}, k \leq s_{2}$} & $(j, k)$ & $(l, m)$ & $P_{D}\left(j-l, S_{2}-m\right)$ & $l>0$ \\
\hline & $(j, k)$ & $(l, m)$ & $P_{D}\left(j-l, S_{2}-m\right)$ & $l \leq 0, m<0$ \\
\hline & $(j, k)$ & $(0, m)$ & $\sum_{w=0}^{w=S_{2}-m} P_{D}\left(j+w, S_{2}-m-w\right)$ & $m \geq 0$ \\
\hline & $(j, k)$ & $(l, 0)$ & $\sum_{w=0}^{w=S_{2}} P_{D}\left(j-l+w, S_{2}-w\right)$ & $l<0$ \\
\hline \multirow{4}{*}{$j \leq s_{1}, k \leq s_{2}$} & $(j, k)$ & $(l, m)$ & $P_{D}\left(S_{1}-l, S_{2}-m\right)$ & $l>0$ \\
\hline & $(j, k)$ & $(l, m)$ & $P_{D}\left(S_{I^{-}}-l, S_{2}-m\right)$ & $l \leq 0, m<0$ \\
\hline & $(j, k)$ & $(0, m)$ & $\sum_{w=0}^{w=S_{2}-m} P_{D}\left(S_{1}+w, S_{2}-m-w\right)$ & $m \geq 0$ \\
\hline & $(j, k)$ & $(l, 0)$ & $\sum_{w=0}^{w=S_{2}} P_{D}\left(S_{1}-l+w, S_{2}-w\right)$ & $l<0$ \\
\hline
\end{tabular}

Table 3: Transition probabilities for an $(R, S, S)$ policy with one-way substitution

$\mathrm{E}\left[\mathrm{B}_{\mathrm{i}}\right]$ can be determined analogously:

$$
E\left[B_{1}\right]=\sum_{j=L B_{o 1}}^{j=-1}\left(-j \sum_{k=L B_{2}}^{k=S_{2}} \pi_{j, k}\right)
$$


$E\left[B_{2}\right]=\sum_{k=L B_{02}}^{k=-1}\left(-k \sum_{j=L B_{1}}^{j=S_{1}} \pi_{j, k}\right)$

The expected order size $E\left[Q_{i}\right]$ follows from

$E\left[Q_{1}\right]=\sum_{j=L B_{1}}^{j=s_{1}} \sum_{k=L B_{2}}^{k=S_{2}}\left(S_{1}-j\right) \pi_{j, k}$

$E\left[Q_{2}\right]=\sum_{j=L B_{1}}^{j=S_{1}} \sum_{k=L B_{2}}^{k=S_{2}}\left(S_{2}-k\right) \pi_{j, k}$

The expected rerouted demand of product 1 fulfilled by product 2 is more complex to calculate. In general, it equals the minimum of the net inventory of product 2 that is leftover after demand of product 2 has been fulfilled, and the demand of product 1 that cannot be fulfilled by its dedicated stock. Moreover, rerouted demand cannot be negative. This yields:

$E[Z]=$

$\sum_{j=L B_{1}}^{j=s_{1}} \sum_{k=L B_{2}}^{k=s_{2}} \pi_{j, k}\left(\sum_{d_{1}=0}^{d_{1}=+\infty} \sum_{d_{2}=0}^{d_{2}=+\infty} P_{D}\left(d_{1}, d_{2}\right) * \max \left\{\min \left\{S_{2}-d_{2}, d_{1}-S_{1}\right\}, 0\right\}\right)+$

$\sum_{j=L B_{1}}^{j=s_{1}} \sum_{k=S_{2}+1}^{k=S_{2}} \pi_{j, k}\left(\sum_{d_{1}=0}^{d_{1}=+\infty} \sum_{d_{2}=0}^{d_{2}=+\infty} P_{D}\left(d_{1}, d_{2}\right) * \max \left\{\min \left\{k-d_{2}, d_{1}-S_{1}\right\}, 0\right\}\right)+$

$\sum_{j=s_{1}+1}^{j=S_{1}} \sum_{k=L B_{2}}^{k=s_{2}} \pi_{j, k}\left(\sum_{d_{1}=0}^{d_{1}=+\infty} \sum_{d_{2}=0}^{d_{2}=+\infty} P_{D}\left(d_{1}, d_{2}\right) * \max \left\{\min \left\{S_{2}-d_{2}, d_{1}-j\right\}, 0\right\}\right)+$

$\sum_{j=S_{1}+1}^{j=S_{1}} \sum_{k=S_{2}+1}^{k=S_{2}} \pi_{j, k}\left(\sum_{d_{1}=0}^{d_{1}=+\infty} \sum_{d_{2}=0}^{d_{2}=+\infty} P_{D}\left(d_{1}, d_{2}\right) * \max \left\{\min \left\{k-d_{2}, d_{1}-j\right\}, 0\right\}\right)$

Alternatively, $E[Z]$ can be obtained from $E\left[d_{i}\right]$ and $E\left[Q_{i}\right]$ :

$E[Z]=E\left[d_{1}\right]-E\left[Q_{1}\right]=E\left[Q_{2}\right]-E\left[d_{2}\right]$

\subsection{DTMC for the $(R, S)$ policy}

The $(R, S)$ policy is a special case of the $(R, S, S)$ policy: at the start of every review period, the inventory level is replenished to level $S_{i}$ (hence, $s_{i}=S_{i}-1$ ). Based on the expressions in Section 3.1, it is now straightforward to formulate the transition probabilities for an $(R, S)$ policy. These are shown in Table 4

\begin{tabular}{|c|c|c|c|}
\hline From & To & Transition probability & For \\
\hline$(j, k)$ & $(l, m)$ & $P_{D}\left(S_{I}-l, S_{2}-m\right)$ & $l>0$ \\
\hline$(j, k)$ & $(l, m)$ & $P_{D}\left(S_{1}-l, S_{2}-m\right)$ & $l \leq 0, m<0$ \\
\hline$(j, k)$ & $(0, m)$ & $\sum_{w=0}^{w=S_{2}-m} P_{D}\left(S_{1}+w, S_{2}-m-w\right)$ & $m \geq 0$ \\
\hline$(j, k)$ & $(l, 0)$ & $\sum_{w=0}^{w=S_{2}} P_{D}\left(S_{1}-l+w, S_{2}-w\right)$ & $l<0$ \\
\hline
\end{tabular}

Table 4: Transition probability of a $(R, S)$ policy for the one-way substitution strategy 
Note that for the $(R, S)$ model with one-way substitution, $\pi_{j, k}$ equals the transition probability to state $(j, k)$ (similar results apply for the no pooling and full pooling strategies). Consequently, the steady state probabilities are readily available from the demand distribution. $E\left[Q_{i}\right], E\left[I_{i}\right], E\left[B_{i}\right]$ and $E[Z]$ can then be reformulated:

$$
\begin{aligned}
& E\left[I_{1}\right]=\sum_{d_{1}=0}^{d_{1}=+\infty} \sum_{d_{2}=0}^{d_{2}=+\infty} \max \left\{S_{1}-d_{1}, 0\right\} P_{D}\left(d_{1}, d_{2}\right) \\
& E\left[I_{2}\right]=\sum_{d_{1}=0}^{d_{1}=+\infty} \sum_{d_{2}=0}^{d_{2}=+\infty} \max \left\{S_{2}-d_{2}-\max \left\{d_{1}-S_{1}, 0\right\}, 0\right\} P_{D}\left(d_{1}, d_{2}\right) \\
& E\left[B_{1}\right]=\sum_{d_{1}=0}^{d_{1}=+\infty} \sum_{d_{2}=0}^{d_{2}=+\infty} \max \left\{d_{1}-S_{1}-\max \left\{S_{2}-d_{2}, 0\right\}, 0\right\} P_{D}\left(d_{1}, d_{2}\right) \\
& E\left[B_{2}\right]=\sum_{d_{1}=0}^{d_{1}=+\infty} \sum_{d_{2}=0}^{d_{2}=+\infty} \max \left\{d_{2}-S_{2}, 0\right\} P_{D}\left(d_{1}, d_{2}\right) \\
& E\left[Q_{1}\right]=\sum_{d_{1}=0}^{d_{1}=+\infty} \sum_{d_{2}=0}^{d_{2}=+\infty}\left(d_{1}-\max \left\{\min \left\{S_{2}-d_{2}, d_{1}-S_{1}\right\}, 0\right\}\right) P_{D}\left(d_{1}, d_{2}\right) \\
& E\left[Q_{2}\right]=\sum_{d_{1}=0}^{d_{1}=+\infty} \sum_{d_{2}=0}^{d_{2}=+\infty}\left(d_{2}+\max \left\{\min \left\{S_{2}-d_{2}, d_{1}-S_{1}\right\}, 0\right\}\right) P_{D}\left(d_{1}, d_{2}\right) \\
& E[Z]=\sum_{d_{1}=0}^{d_{1}=+\infty} \sum_{d_{2}=0}^{d_{2}=+\infty} \max \left\{\min \left\{S_{2}-d_{2}, d_{1}-S_{1}\right\}, 0\right\} P_{D}\left(d_{1}, d_{2}\right)=E\left[d_{1}\right]-E\left[Q_{1}\right]=E\left[Q_{2}\right]-E\left[d_{2}\right]
\end{aligned}
$$

\section{Numerical experiments}

In this section, we determine the optimal inventory control parameters (and corresponding expected total costs) for a one-way substitution system with a given set of cost parameters and different demand correlations. The results are compared to the optima of the no pooling and full pooling strategies (the details of the DTMC approach for these two strategies can be found in Appendix A and B respectively).

\subsection{Experimental setting}

Table 5 gives an overview of the parameter values used in the experiments. The demand for both product types is assumed to follow a (discretized) bivariate normal demand distribution, based on a joint continuous normal distribution $\mathrm{f}(\mathrm{x}, \mathrm{y}) \sim \mathrm{N}_{2}(\mu, \Sigma)$ with mean vector $\mu=[20,20]$ and covariance matrix $\Sigma=$

$9 *\left[\begin{array}{ll}1 & \rho \\ \rho & 1\end{array}\right]$ (the details of the discretization procedure can be found in Appendix C). The demand correlation $\rho$ is varied $(\rho=-0.9,0$ or 0.9$)$ to study its influence on the optimal inventory control parameters and the expected total cost. Note that the flexible product (i.e. product 2) has a $10 \%$ product cost premium over product 1 .

We apply holding cost rates of $25 \%$ of the unit purchasing cost for both product types. The unit penalty cost for not satisfying demand is equal to 2 euro for both products. The adjustment cost is 0.2 euro per 
unit of rerouted demand. Note that these cost parameters satisfy assumptions 1,2 and 3 discussed in Section 2.

\begin{tabular}{|l|c|c|}
\hline & Product 1 & Product 2 \\
\hline $\boldsymbol{E}\left[\boldsymbol{d}_{\boldsymbol{i}}\right]$ & 20 & 20 \\
\hline $\boldsymbol{\sigma}\left[\boldsymbol{d}_{\boldsymbol{i}}\right]$ & 3 & 3 \\
\hline Correlation $\boldsymbol{\rho}$ & \multicolumn{2}{|c|}{$-0.9 ; 0 ; 0.9$} \\
\hline $\boldsymbol{c}_{\boldsymbol{i}}$ & 4 & 4.4 \\
\hline $\boldsymbol{h}_{\boldsymbol{i}}$ & 1 & 1.1 \\
\hline $\boldsymbol{p}_{\boldsymbol{i}}$ & 2 & 2 \\
\hline $\boldsymbol{a}$ & 0.2 & - \\
\hline
\end{tabular}

Table 5: Parameter values

\subsection{Results for the $(R, S)$ policy}

Table 6 gives an overview of the optimal inventory control parameters $\left(S_{i}^{*}\right)$ and the expected cost components for the three strategies, assuming both items are managed according to an $(\mathrm{R}, \mathrm{S})$ policy. The optimal values for $S_{i}$ were found using a steepest descent algorithm ${ }^{3}$.

The one-way substitution strategy outperforms both the no pooling strategy and the full pooling strategy, resulting in a lower optimal expected total cost irrespective of the correlation. Note that, at high correlation, the optimal $S_{i}$ of the substitution scenario coincide with those of the no pooling scenario, while the difference in total cost becomes very small. The reason is that, at high correlation, the option to reroute demand is seldom used (as evident from the very small value for adjustment $\operatorname{cost}^{4}$ ), as high demand for product 1 tends to go hand in hand with high demand for product 2, resulting in a higher probability of simultaneous depletion of both stocks. Nevertheless, the mere opportunity to use of this option results in slight changes to the remaining cost components: the shortage cost for product 1 decreases, along with its purchasing cost. The holding cost for product 2 also decreases, while the purchasing cost increases. Due to assumption 2 discussed in Section 3, the net effect of the rerouting option is positive.

\footnotetext{
${ }^{3}$ As the objective function for the (R,S) policy is provably convex (see Deflem and Van Nieuwenhuyse 2011), the steepest descent algorithm is guaranteed to converge to the global optimum.

${ }^{4}$ This can be seen even more clearly by investigating $E[Z]$ in Table 7 .
} 


\begin{tabular}{|c|c|c|c|c|c|c|}
\hline \multirow{2}{*}{ Correlation } & \multirow{2}{*}{$\begin{array}{c}\text { Expected cost } \\
\text { per period }\end{array}$} & \multicolumn{2}{|c|}{ No pooling } & Full pooling & \multicolumn{2}{|c|}{ One-way substitution } \\
\hline & & Product 1 & Product 2 & Product 2 & Product 1 & Product 2 \\
\hline \multirow{6}{*}{0,9} & $S_{i}^{*}$ & 21 & 21 & \multirow{6}{*}{$\begin{array}{l}2 \\
3,815 \\
2,936 \\
176 \\
4 \\
\quad \underline{186,751}\end{array}$} & 21 & 21 \\
\hline & Holding cost & 1,758 & 1,933 & & 1,758 & 1,908 \\
\hline & Backlog cost & 1,515 & 1,515 & & 1,468 & 1,515 \\
\hline & & 80 & 88 & & 79,907 & 88,103 \\
\hline & \multirow{2}{*}{$\begin{array}{l}\text { Adjustment cost } \\
\underline{\text { Total cost }}\end{array}$} & - & - & & 0,005 & - \\
\hline & & \multicolumn{2}{|c|}{$\underline{174,721}$} & & \multicolumn{2}{|c|}{174,664} \\
\hline \multirow{6}{*}{ 0 } & $S_{i}^{*}$ & 21 & 21 & \multirow{6}{*}{ 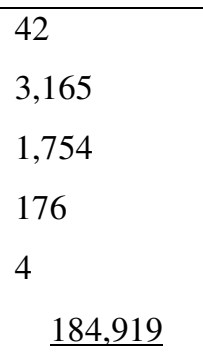 } & 20 & 22 \\
\hline & Holding cost & 1,758 & 1,933 & & 1,191 & 2,013 \\
\hline & Backlog cost & 1,515 & 1,515 & & 1,145 & 0,898 \\
\hline & Purchasing cost & 80 & 88 & & 77,525 & 90,723 \\
\hline & Adjustment cost & - & - & & 0,124 & - \\
\hline & $\underline{\text { Total cost }}$ & \multicolumn{2}{|c|}{$\underline{174,721}$} & & \multicolumn{2}{|c|}{$\underline{173,619}$} \\
\hline \multirow{6}{*}{$-0,9$} & $S_{i}^{*}$ & 21 & 21 & \multirow{6}{*}{$\begin{array}{l}41 \\
1,295 \\
0,355 \\
176 \\
4 \\
\quad \underline{181,650}\end{array}$} & 18 & 23 \\
\hline & Holding cost & 1,758 & 1,933 & & 0,449 & 1,034 \\
\hline & Backlog cost & 1,515 & 1,515 & & 0,285 & 0,493 \\
\hline & Purchasing cost & 80 & 88 & & 70,774 & 98,149 \\
\hline & \multirow{2}{*}{$\begin{array}{c}\text { Adjustment cost } \\
\underline{\text { Total cost }}\end{array}$} & - & - & & 0,461 & \\
\hline & & \multicolumn{2}{|c|}{$\underline{174,721}$} & & \multicolumn{2}{|c|}{171,645} \\
\hline
\end{tabular}

Table 6: Overview of optimal $S_{i}^{*}$ and optimal expected costs for the $(R, S)$ policy

Table 6 also shows that because of the risk-pooling effect, decreasing the demand correlation improves the optimal cost for both the full pooling and the one-way substitution strategy. Note that the optimal order-up-to levels in the one-way substitution strategy diverge more as correlation decreases: this is intuitive, as a lower correlation increases the attractiveness of pooling demands on the flexible item. Consequently, S1 decreases, and S2 increases. The demand correlation has no influence on the optimal inventory control parameters and expected costs for the no pooling strategy: this is intuitive, as there is no interaction between both inventories.

Table 7 shows the associated values for $E\left[I_{i}\right], E\left[B_{i}\right], E\left[Q_{i}\right]$, and $E[Z]$ of the different scenarios. The total optimal safety stock (or leftover inventory at the end of the review period) is the lowest for the full pooling strategy and the highest for the no pooling strategy. The reason is straightforward. When the full pooling strategy is used, the total demand for both products is pooled (observe the expected amount rerouted is equal to the average demand of product 1). With the one-way substitution strategy, only part of the demand for product 1 is rerouted to the substitute (see $E[Z]$ ) resulting in a smaller safety stock 
reduction. In addition, Table 7 shows that reducing demand correlation results in rerouting more demand to the substitute in case of a one-way substitution strategy. As a consequence, the amount purchased of product 1 decreases, the amount purchased of product 2 increases and the total need for safety stock reduces.

\begin{tabular}{|c|c|c|c|c|c|c|}
\hline \multirow{2}{*}{ Correlation } & & \multicolumn{2}{|c|}{ No pooling } & \multirow{2}{*}{$\begin{array}{c}\text { Full pooling } \\
\text { Product } 2\end{array}$} & \multicolumn{2}{|c|}{ One-way substitution } \\
\hline & & Product 1 & Product 2 & & Product 1 & Product 2 \\
\hline \multirow{5}{*}{0,9} & $S_{i}^{*}$ & 21 & 21 & 42 & 21 & 21 \\
\hline & $E\left[I_{i}\right]$ & 1,758 & 1,758 & 3,468 & 1,758 & 1,734 \\
\hline & $E\left[B_{i}\right]$ & 0,758 & 0,758 & 1,468 & 0,734 & 0,758 \\
\hline & $E\left[Q_{i}\right]$ & 20 & 20 & 40 & 19,977 & 20,023 \\
\hline & $E[Z]$ & - & _- & 20 & 0,0234 & - \\
\hline \multirow{5}{*}{$\mathbf{0}$} & $S_{i}^{*}$ & 21 & 21 & 42 & 20 & 22 \\
\hline & $E\left[I_{i}\right]$ & 1,758 & 1,758 & 2,877 & 1,191 & 1,830 \\
\hline & $E\left[B_{i}\right]$ & 0,758 & 0,758 & 0,877 & 0,572 & 0,449 \\
\hline & $E\left[Q_{i}\right]$ & 20 & 20 & 40 & 19,381 & 20,619 \\
\hline & $E[Z]$ & - & - & 20 & 0,619 & - \\
\hline \multirow{5}{*}{$-0,9$} & $S_{i}^{*}$ & 21 & 21 & 41 & 18 & 23 \\
\hline & $E\left[I_{i}\right]$ & 1,758 & 1,758 & 1,177 & 0,449 & 0,940 \\
\hline & $E\left[B_{i}\right]$ & 0,758 & 0,758 & 0,177 & 0,142 & 0,247 \\
\hline & $E\left[Q_{i}\right]$ & 20 & 20 & 40 & 17,693 & 22,307 \\
\hline & $E[Z]$ & _ & _ & 20 & 2,307 & _ \\
\hline
\end{tabular}

Table 7: Overview of optimal $E\left[I_{i}\right], E\left[B_{i}\right], E\left[Q_{i}\right]$ and $E[Z]$ for the $(R, S)$ policy

\subsection{Results for the $(R, s, S)$ policy}

Pending a convexity proof for the $(\mathrm{R}, \mathrm{s}, \mathrm{S})$ policy with zero leadtimes, we used exhaustive search to detect the optimal $s_{i}$ and $S_{i}$ for the different scenarios. The results are shown in Table 8 .

The most remarkable observation is that the optimal $(R, S, S)$ policy coincides with the optimal $(R, S)$ policy for every scenario (i.e. $\left.s_{i}^{*}=S_{i}^{*}-1\right)^{5}$. Consequently, the expected cost components for the $(R, S)$ policy are the same as those observed for the $(R, s, S)$ policy (as shown previously in Table 7 ), and the insights

\footnotetext{
${ }^{5}$ Due to the discretization procedure, the $\mathrm{s}_{\mathrm{i}}{ }^{*}$ values for any given $\mathrm{S}_{\mathrm{i}}^{*}$ are in fact not unique: for all scenarios, alternative optimal solutions were found at $\mathrm{s}_{\mathrm{i}}{ }^{*}$ values strictly smaller than $\mathrm{S}_{\mathrm{i}}{ }^{*}-1$. This is due to the fact that demand is limited by lower bound values strictly larger than 1 for each product type (as discussed in Appendix C), implying that the minimum observable demand is at least equal to the lower bound. As this outcome is an artifact of the discretization procedure, the alternative optima are not mentioned in the table.
} 
discussed in Section 4.2 remain valid. We expect that this striking result is due to the fact that our objective function does not account for fixed order costs; consequently, there is no penalty for ordering frequently and the decision maker has an incentive to replenish stock as soon as possible (which implies $s=S-1$, and hence the two policies coincide).

\begin{tabular}{|c|c|c|c|c|c|c|}
\hline \multirow{2}{*}{ Correlation } & \multirow{2}{*}{$\begin{array}{c}\text { Expected cost } \\
\text { per period }\end{array}$} & \multicolumn{2}{|c|}{ No pooling } & Full pooling & \multicolumn{2}{|c|}{ One-way substitution } \\
\hline & & Product 1 & Product 2 & Product 2 & Product 1 & Product 2 \\
\hline \multirow{7}{*}{0,9} & $s_{i}^{*}$ & 20 & 20 & \multirow{7}{*}{ 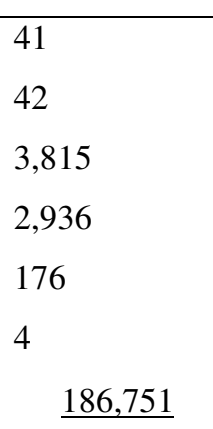 } & 20 & 20 \\
\hline & $S_{i}^{*}$ & 21 & 21 & & 21 & 21 \\
\hline & Holding cost & 1,758 & 1,933 & & 1,758 & 1,908 \\
\hline & Backlog cost & 1,515 & 1,515 & & 1,468 & 1,515 \\
\hline & Purchasing cost & 80 & 88 & & 79,907 & 88,103 \\
\hline & \multirow{2}{*}{$\begin{array}{l}\text { Adjustment cost } \\
\underline{\text { Total cost }}\end{array}$} & - & - & & 0,005 & - \\
\hline & & \multicolumn{2}{|c|}{174,721} & & \multicolumn{2}{|c|}{174,664} \\
\hline \multirow{7}{*}{$\mathbf{0}$} & $s_{i}^{*}$ & 20 & 20 & \multirow{7}{*}{ 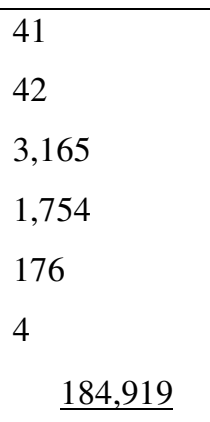 } & 19 & 21 \\
\hline & $S_{i}{ }^{*}$ & 21 & 21 & & 20 & 22 \\
\hline & Holding cost & 1,758 & 1,933 & & 1,191 & 2,013 \\
\hline & Backlog cost & 1,515 & 1,515 & & 1,145 & 0,898 \\
\hline & Purchasing cost & 80 & 88 & & 77,525 & 90,723 \\
\hline & Adjustment cost & - & - & & 0,124 & - \\
\hline & $\underline{\text { Total cost }}$ & \multicolumn{2}{|c|}{174,721} & & \multicolumn{2}{|c|}{$\underline{173,619}$} \\
\hline \multirow{7}{*}{$-0,9$} & $s_{i}^{*}$ & 20 & 20 & \multirow{7}{*}{$\begin{array}{l}40 \\
41 \\
1,295 \\
0,355 \\
176 \\
4 \\
\\
\underline{181,650} \\
\end{array}$} & 17 & 22 \\
\hline & $S_{i}^{*}$ & 21 & 21 & & 18 & 23 \\
\hline & Holding cost & 1,758 & 1,933 & & 0,449 & 1,034 \\
\hline & Backlog cost & 1,515 & 1,515 & & 0,285 & 0,493 \\
\hline & Purchasing cost & 80 & 88 & & 70,774 & 98,149 \\
\hline & Adjustment cost & - & - & & 0,461 & - \\
\hline & $\underline{\text { Total cost }}$ & \multicolumn{2}{|c|}{$\underline{174,721}$} & & \multicolumn{2}{|c|}{171,645} \\
\hline
\end{tabular}

Table 8: Overview of optimal $s_{i}^{*}, S_{i}^{*}$ and optimal expected costs for the $(R, s, S)$ policy

\section{Conclusions}

In this paper we studied the effect of the one-way substitution strategy on the optimal design of a twoitem inventory system using an $(R, S, S)$ or $(R, S)$ replenishment policy with negligible leadtimes. We present a discrete time Markov model to evaluate the expected total cost function for any arbitrary set of 
inventory control parameters. Numerical results show that the one-way substitution strategy can outperform both the no pooling and full pooling strategies even when the cost premium for flexibility is significant. Furthermore, we can observe that decreasing the demand correlation results in rerouting more demand to the flexible product; because of the risk-pooling effect, this reduces the optimal expected total cost. For the current experiments, the optimal $(R, S, S)$ policy coincides with the optimal $(R, S)$ policy, though we expect this to change once fixed order costs are taken into account in the objective function. Future research will focus primarily on including positive (possibly stochastic) replenishment leadtimes in the analysis; this issue is highly relevant to adequately represent real-life settings.

\section{References}

Bassok Y., R. Anupindi and R. Akella (1999). Single-period multiproduct inventory models with substitution. Operations Research 47, 632-642.

Chopra S. and Meindl P. (2007). Supply Chain Management: Strategy, planning \& Operation, third edition. Pearson Prentice Hall, New Jersey.

Deflem Y. and Van Nieuwenhuyse I. (2011). Evaluating the optimal design of a two-item periodic inventory system with one-way substitution: a newsvendor approach. FBE Research Report, K.U.Leuven - Faculty of Business and Economics, forthcoming.

Hale W., D. Pyke and N. Rudi, (2001). An assemble-to-order system with component substitution. Working Paper, Tuck School of Business.

Herer Y.T., M. Tzur and E. Yücesan (2006). The multilocation transshipment problem. IIE Transactions 38, 185-200.

Hillier M. S. (2002). Using commonality as backup safety stock. European Journal of Operational Research 136, 353-365.

Khouja M., A. Mehrez and G. Rabinowitz (1996). A two-item newsboy problem with substitution. International Journal of production Economics 44, 267-275. 
Robinson L.W. (1990). Optimal and approximate policies in multiperiod, multilocation inventory models with transshipments. Operations research 38, 278-295.

Rutten W.G.M.M. and Bertrand (1998). Balancing stocks, flexible recipe costs and high service level requirements in a batch process industry: A study of a small scale model. European Journal of Operational Research 110, 626-642.

Tibben-Lembke R.S. and Y. Bassok (2005). An inventory model for delayed customization: A hybrid approach. European Journal of Operational Research 165, 748-764.

Van Mieghem J. A. (1998). Investment Strategies for Flexible Resources. Management Science 44, 10711078.

Van Mieghem J. A. (2008), Operations Strategy: Principles and Practice, Dynamic Ideas, Belmont

Van Mieghem J. A. and N. Rudi (2002). Newsvendor networks: Inventory management and capacity investment with discretionary activities. Manufacturing \& Service Operations Management 4, 313-335. 


\section{Appendix A}

As substitution cannot occur in the no pooling strategy, the inventories of both products can be analyzed separately, yielding two one-dimensional DTMCs. Tables A.1 and A.2 present the transition probabilities for the $(\mathrm{R}, \mathrm{s}, \mathrm{S})$ policy and $(\mathrm{R}, \mathrm{S})$ policy respectively. Note, $P_{D 1}\left(d_{1}\right)$ denotes the marginal probability mass function of demand for product 1 and is defined as $P_{D 1}\left(d_{1}\right)=\sum_{d_{2}} P_{D}\left(d_{1}, d_{2}\right)$. The marginal probability mass function of demand for product $2\left(P_{D 2}\left(d_{2}\right)\right)$ is defined analogously.

The expected rerouted demand equals zero (since demand can only be fulfilled from the dedicated inventory). The remaining objective function components for the no pooling and full pooling strategies can be calculated in a way analogous to the approach discussed in Section 3.1. Note that the lower bound on the net inventory of product $i$ for the no pooling strategy is $L B_{i}=s_{i}+1-\max \left(d_{i}\right)$.

\begin{tabular}{|c|c|c|c|}
\hline Product 1 & From & To & Transition probability \\
\hline$j>s_{1}$ & $(j)$ & $(l)$ & $P_{D 1}(j-l)$ \\
\hline$j \leq s_{1}$ & $(j)$ & $(l)$ & $P_{D 1}\left(S_{1}-l\right)$ \\
\hline Product 2 & From & To & Transition probability \\
\hline$k>s_{2}$ & $(k)$ & $(m)$ & $P_{D 2}(k-m)$ \\
\hline$k \leq s_{2}$ & $(k)$ & $(m)$ & $P_{D 1}\left(S_{2}-m\right)$ \\
\hline
\end{tabular}

Table A.1: Transition probabilities for an $(R, s, S)$ policy with no pooling

\begin{tabular}{|c|c|c|c|}
\hline Product 1 & From & To & Transition probability \\
\hline & $(j)$ & $(l)$ & $P_{D 1}\left(S_{1}-l\right)$ \\
\hline Product 2 & From & To & Transition probability \\
\hline & $(k)$ & $(m)$ & $P_{D 1}\left(S_{2}-m\right)$ \\
\hline
\end{tabular}

Table A.2: Transition probabilities for an $(R, S)$ policy with no pooling 


\section{Appendix B}

In case of the full pooling strategy, only inventory of product 2 is kept. We model the system using a onedimensional DTMC 6 . Tables B.1 and B.2 present the transition probabilities for the $(\mathrm{R}, \mathrm{s}, \mathrm{S})$ policy and $(\mathrm{R}, \mathrm{S})$ policy respectively.

The expected rerouted demand equals the expected demand of product 1 over the review period (as all demand of product 1 is rerouted to product 2, and all unmet demand is fully backlogged). The remaining objective function components for full pooling strategies can also be calculated in a way analogous to the approach discussed in Section 3.1. Note that the lower bound on the net inventory of product 2 is $L B_{2}=s_{2}+1-\max \left(d_{1}\right)-\max \left(d_{2}\right)$.

\begin{tabular}{|c|c|c|c|}
\hline & From & To & Transition probability \\
\hline$k>s_{2}$ & $(k)$ & $(m)$ & $\sum_{w=0}^{k-m} P_{D}(w, k-m-w)$ \\
\hline$k \leq s_{2}$ & $(k)$ & $(m)$ & $\sum_{w=0}^{S_{2}-m} P_{D}\left(w, S_{2}-m-w\right)$ \\
\hline
\end{tabular}

Table B.1: Transition probabilities of product 2 for an $(R, s, S)$ policy with full pooling

\begin{tabular}{|c|c|c|}
\hline From & To & Transition probability \\
\hline$(k)$ & $(m)$ & $\sum_{w=0}^{S_{2}-m} P_{D}\left(w, S_{2}-m-w\right)$ \\
\hline
\end{tabular}

Table B.2: Transition probabilities of product 2 for an $(R, S)$ policy with full pooling

\footnotetext{
${ }^{6}$ Note that this approach is only applicable when the penalty costs for both products are equal (as in our numerical experiments presented in section 4.2 and 4.3), since we do not keep track of the backorder positions for product 1 and 2 separately.
} 


\section{Appendix C}

In this section we illustrate the discretization procedure of the continuous bivariate normal demand distribution with probability density function $f(x, y)$. The discrete joint probability mass function $P_{D}\left(d_{1}, d_{2}\right)$ is then obtained from

$$
P_{D}\left(d_{1}, d_{2}\right)=\int_{d_{1}-0,5}^{d_{1}+0,5} \int_{d_{2}-0,5}^{d_{2}+0,5} f(x, y) d y d x
$$

As we assume that product demands are finite, we limit the discrete demand vectors $\left(\mathrm{d}_{1}, \mathrm{~d}_{2}\right)$ between a lower $\left(\mathrm{L}_{1}, \mathrm{~L}_{2}\right)$ and upper boundary $\left(\mathrm{U}_{1}, \mathrm{U}_{2}\right)$. Demand vectors outside these boundaries obtain $P_{D}\left(d_{1}, d_{2}\right)=$ 0 in the final discrete demand distribution; for demand vectors that lie between the boundaries, the final $P_{D}\left(d_{1}, d_{2}\right)$ is rescaled to ensure that $\sum_{d 2=L 2}^{d 2=U 2} \sum_{d 1=L 1}^{d 1=U 1} P_{D}\left(d_{1}, d_{2}\right)=1$.

Table C.1 gives an overview of the boundaries implemented in the numerical experiments of Section 4 , where $\mathrm{f}(\mathrm{x}, \mathrm{y}) \sim \mathrm{N}_{2}(\mu, \Sigma)$ with mean vector $\mu=[20,20]$ and covariance matrix $\Sigma=9 *\left[\begin{array}{ll}1 & \rho \\ \rho & 1\end{array}\right]$. The demand correlation $\rho$ is varied $(\rho=-0.9,0$ or 0.9$)$

\begin{tabular}{|l|l|l|}
\hline Correlation & Lower boundary & Upper boundary \\
\hline $\mathbf{- 0 , 9}$ & $(4,4)$ & $(36,36)$ \\
\hline $\mathbf{0}$ & $(7,7)$ & $(33,33)$ \\
\hline $\mathbf{0 , 9}$ & $(4,4)$ & $(36,36)$ \\
\hline
\end{tabular}

Table C.1: Boundaries of the discrete joint probability mass functions used in Section 4 